\title{
Bioactive microfluidic paper device for pesticide determination
}

\section{in waters}

M.D. Fernández-Ramos ${ }^{* a, c}$, A.L. Ogunneye ${ }^{\mathrm{b}}$, N.A.A.Barbarinde ${ }^{\mathrm{b},}$ M.M. Erenas ${ }^{\mathrm{a}, \mathrm{c}}$, L.F. Capitán-Vallvey ${ }^{\mathrm{a}, \mathrm{c}}$

${ }^{a}$ Department of Analytical Chemistry, Campus Fuentenueva, Faculty of Sciences, 18071, University of Granada, Spain.

${ }^{b}$ Department of Chemical Sciences, Olabisi Onabanjo University, Ago-Iwoye, Ogun State, Nigeria.

${ }^{c}$ Unit of Excellence in Chemistry applied to Biomedicine and the Environment of the University of Granada, Spain.

*e-mail: mdframos@ugr.es

\begin{abstract}
This work presents a new optical microfluidic paper biosensor for the detection of organophosphate pesticides and carbamate pesticides. The assay strip is composed of a paper support $(1 \times 17.6 \mathrm{~mm})$ onto which acetylcholine esterase $(\mathrm{AChE})$ and acetylcholine chloride $(\mathrm{AChCl})$ are deposited, in such a way that there is a small hole between them that ensures that they only come into contact in the reaction zone when they are carried by a solution of the sample by lateral flow to the reaction zone containing bromocresol purple (BCP) as the $\mathrm{pH}$ indicator, immobilized by sol-gel. The sensor operates at room temperature and the rate of the inhibited reaction serves as an analytical signal, which is measured using a camera by quantifying the appropriate colour coordinate. Calibration curves were obtained for chlorpyrifos and carbaryl, with a useful concentration range from 0.24 to $20 \mu \mathrm{g} \cdot \mathrm{L}^{-1}$ for carbaryl and from 2.00 to $45 \mu \mathrm{g} \cdot \mathrm{L}^{-1}$ for chlorpyrifos. The detection limits were 0.24 and $2.00 \mu \mathrm{g} \cdot \mathrm{L}^{-1}$, respectively, and with reproducibility around 4.2-5.5\%. The method was applied to the determination of pesticides in different water samples, with no sample preparation.
\end{abstract}

Keywords: Microfluidic paper sensor; biosensor; pesticides; organophosphate and carbamate pesticides, water samples

* Corresponding author; e-mail: mdframos@ugr.es 


\section{Introduction}

Modern agriculture has a high productive capacity that defines it as an activity designed to respond to market needs and to sell thousands of tonnes of products both domestically and internationally. To achieve this, it is necessary to use pesticides that protect crops in order to prevent, destroy or control the pests, in the form of animals or plants, that interfere with the crop production chain.

The use of pesticides has become more intensified in agriculture, leading to the risk of their accumulation in soil, agrofood products, and water, resulting in environmental pollution that is harmful to living organisms and human health [1]. Even at low levels, pesticide residues can seriously affect the nervous system of living organisms once they enter the food chain[2]. The inhibition of the acetylcholinesterase enzyme (AChE) result in the accumulation of the neurotransmitter acetylcholine in the body leading to organ failure [3]. There is, therefore, a real need to effectively and economically detect and monitor the level of pesticides in the environment and agricultural products. The detection of pesticides is of great interest, thus the European Union has established the maximum residue level (MRL) of permitted pesticides in the range of $0.01-0.02 \mathrm{mg} \cdot \mathrm{L}^{-1}$ for many fruits (https://ec.europa.eu/food/plant/pesticides/eu-pesticides-database/). Moreover, pesticide residues are concentrated in rivers and ponds, via rain or irrigation runoff, near locations where there is an extensive use of pesticides, originating water pollution. Thus, an simple, portable and easy to use analytical devise for pesticides determination is still required.

The current laboratory-based methods for pesticide analysis include GC[4], HPLC [5], GC/HPLC-MS[6] and CE[7]. These methods have very good analytical characteristics, but they are not suitable for on-site and/or real-time analysis because they require a laboratory, have a relatively long and complex assay time, costly instrumentation and maintenance and require a qualified technician. However, the recent emergence of microfluidic devices in the field of analytical chemistry has led to a revolution in combining microfabrication, electronics and chemistry with the aim of putting simple environmentally-friendly analytical tools into the hands of the public to use anywhere, with a fast response and high selectivity either using simple low-cost instruments or even an instrument-free technique.

One type of analytical devices of interest are capillary devices, largely based on paper, because of the advantages with regard to cost, their straightforward manufacturing 
process, easy functionalization, usefulness for analytical operation implementation and biocompatibility. Various $\mu \mathrm{PAD}$ devices have been proposed for the determination of pesticides based on different chemicals and detection methods, mainly colorimetric, luminescent and electrochemical [8]. In colorimetric detection, a device has been described to determine trichlorfon residues based on colorimetric phosphorus detection like molybdenum blue after UV irradiation [9]. One interesting approach for the determination of 2,4D uses CdTe QDs deposited on paper cellulose fibres with MIPs built in, with quenching by electron-transfer-induced mechanism for 2,4D[10]. However, most of the proposed $\mu$ PADs are based on the inhibition of acetylcholinesterase (AChE) or butyryl-cholinesterase activity by organophosphate or carbamate pesticides. Different strategies have been proposed based on this inhibition. A 3D device, consisting of two sheets for testing and sampling involving the AChE-catalysed hydrolysis of acetylthiocholine to produce thiocholine causes the aggregation of graphene-quantumdot capped GQD-AuNPs with a colour change, and aggregation that is inhibited by chlorpyrifos[11]. The combination of $\mathrm{AChE}$ and choline oxidase catalyse the formation of $\mathrm{H}_{2} \mathrm{O}_{2}$ from acetylcholine, which is detected by nanoceria-coated paper, producing a yellow colour, except in the presence of pesticides, which reduce the intensity of the yellow[12]. However, these methods require different operating steps that are performed manually, such as adding external reagents or inverting the device at a given time to produce the colour. To solve these problems, a flow control-based 3D $\mu \mathrm{PAD}$ is proposed in which the flow rate is controlled by modifying the length and width of the channels for an optimum interaction between the pesticide, AChE and chromogen indoxyl acetate, which promotes their hydrolysis and subsequent atmospheric oxidation to blue indigo dye[13]. Alternatively, a dual flow channel design has been proposed in which the fluid moves at different speeds by sandwiching the paper channels between flexible films[14]. Another solution to integrate all the steps is to use multilayer paper devices such as the six-layer device proposed by Yang et al. [15].

This study presents a bioactive microfluidic paper device for the determination of organophosphorus pesticides, using chlorpyrifos as a model, and carbamate pesticides, with carbaryl as a model, in waters, using an original and effective paper design as the sensor platform where the enzyme is immobilized. The signal transduction is the result of substrate hydrolysis by the inhibited enzyme, which produces local $\mathrm{pH}$ alterations of the sol-gel entrapped indicator with a colour change that it is measured with a 
photographic camera using colour coordinates as the analytical parameter for the quantification of pesticides.

\section{Experimental section}

\subsection{Materials and equipment}

The filter paper used as the support to make the $\mu \mathrm{PAD}$ was Filter-Lab (www.fanoia.com) ref. 1238 (basis weight $80 \mathrm{~g} \cdot \mathrm{m}^{-2}$; thickness $150 \mu \mathrm{m}$; retention $20-25 \mu \mathrm{m}$ ). Acetylcholinesterase $(\mathrm{AChE})$, acetylcholine chloride $(\mathrm{AChCl})$, bovine serum albumin (BSA), bromocresol purple sodium salt (BP), cresol red, tetraethoxysilane (TEOS), phenyltrimethoxylane (PTMOS), sodium hydrogen phosphate, and sodium dihydrogen phosphate came from Sigma Aldrich Merck (Madrid, Spain). Chloropyriphos Pestanal ${ }^{\circledR}$ and carbetamide Pestanal $^{\circledR}$ came from Riedel de Haën (Germany). All reagents were of analytical reagent grade unless otherwise stated. All aqueous solutions were made using reverse osmosis type quality water (Milli-RO 12 plus Milli-Q station from Millipore, conductivity $18.2 \mathrm{M} \Omega \cdot \mathrm{cm})$.

Digital images from the $\mu \mathrm{PAD}$ were captured using a Canon PowerShot G12 digital camera (Canon Inc., Tokio, Japan). ImageJ (National Institutes of Health) software was used to analyse the region of interest (ROI) of the images. The $\mu \mathrm{PAD}$ was designed using Adobe Illustrator software (Adobe Inc., California, USA) and cut using a Rayjet Trotec Laser engraving printer (Trotec, Austria) with Rayjet Commander software.

\subsection{Solution preparation}

$1 \mathrm{mg} \cdot \mathrm{mL}^{-1}$ solution of bovine serum albumin (BSA) was prepared by dissolving $5 \mu \mathrm{L}$ of $200 \mathrm{mg} \cdot \mathrm{mL}^{-1}$ of BSA in $5 \mathrm{mM}$ phosphate buffer $\mathrm{pH}$ 7.0. AChE stock solution at $5 \mathrm{U} \cdot \mathrm{mL}^{-}$ ${ }^{1}$ was made from $502.74 \mathrm{U} \cdot \mathrm{mg}^{-1}$ of AChE adding BSA solution in $5 \mathrm{mM}$ phosphate buffer $\mathrm{pH}$ 7.0. $\mathrm{AChCl} 15 \mathrm{mM}$ stock solution was made in $5 \mathrm{mM}$ phosphate buffer $\mathrm{pH}$ 7.0. The stock solution was stored at $4^{\circ} \mathrm{C}$ and working $\mathrm{AChCl}$ solutions were prepared daily by diluting the stock solution using $5 \mathrm{mM}$ phosphate buffer $\mathrm{pH}$ 7.0. All stocks solutions were stored at $2-8{ }^{\circ} \mathrm{C}$ until use. The standard solutions of both pesticides, chlorpyrifos and carbaryl, were prepared daily by diluting an appropriate volume of each stock pesticide $\left(1 \mathrm{mg} \cdot \mathrm{mL}^{-1}\right.$ in acetonitrile) with $5 \mathrm{mM}$ phosphate buffer $\mathrm{pH} 7.0$ and stored at $4^{\circ} \mathrm{C}$ until use. 


\subsection{Sol-gel material preparation}

The method used for sol-gel preparation is based on a method proposed by Makote and col. [16] using an acid-catalysed hydrolysis. TEOS and PTMOS (2:1 molar ratio) were mixed to $0.70 \mathrm{~mL}$ water, $0.50 \mathrm{~mL}$ ethanol and $0.35 \mathrm{~mL} 0.1 \mathrm{M} \mathrm{HCl}$ and sonicated for $2 \mathrm{~min}$. After left overnight at room temperature, $0.5 \mathrm{mg}$ of the $\mathrm{pH}$ indicator $\mathrm{BP}$ was added to $1 \mathrm{~mL}$ of prepared sol-gel. The mixture was vortexed for $5 \mathrm{~min}$ to obtain a uniform sol-gel. The vial was kept in the dark and at room temperature until use.

\subsection{Fabrication of the $\mu P A D$}

The devices were prepared in filter paper using a laser cutting technique. The pattern was designed with Adobe Illustrator software and cut with a desktop laser engraver with a 12 $\mathrm{W} \mathrm{CO}_{2}$ laser source. To optimize the procedure and the substrate used, the $\mu$ PAD elements were produced by cutting the plastic-backed cellulose paper and removing the weeding manually with a rate of success of $99 \%$.

Prior to using the paper support, it was cleaned by immersion in purified water and magnetically stirred for 10 minutes. Then, the water was replaced by ethanol and magnetically stirred for another 10 minutes. It was then decanted and the sensors spread on a clean white paper to dry at room temperature. The device (Fig. 1) consists of three separate zones, one for sampling (bottom $\mu \mathrm{PAD}$ ), two transport channels separated by a gap where the $\mathrm{AChE}$ and $\mathrm{AChCl}$ solutions are deposited in each one, and a detection zone containing the $\mathrm{pH}$ indicator in sol-gel (top $\mu \mathrm{PAD}$ ). The device was prepared by dropcasting the needed reagents into each respective zone at room temperature. To prepare the detection zone, $2 \mu \mathrm{L}$ of a solution containing $0.5 \mathrm{mg} \cdot \mathrm{mL}^{-1} \mathrm{BP}$ in sol-gel was dispensed and then dried at room temperature (Fig. 1b). Next, the BP was turned to its alkaline form by immersing the device in $5 \mathrm{mM}$ phosphate buffer $\mathrm{pH} 8.5$ in $100 \mathrm{mM} \mathrm{NaCl}$ for $30 \mathrm{~min}$ with magnetic stirring. After this, the device was dried at room temperature. The transport channel was prepared by adding $0.5 \mu \mathrm{L}$ of $1 \mathrm{U} \cdot \mathrm{mL}^{-1}$ of AChE in the left channel and $1 \mu \mathrm{L}$ of an $8 \mathrm{mM} \mathrm{AchCl}$ solution in the right channel, then dried at room temperature for $5 \mathrm{~min}$. The $\mu$ PADs were stored in a dry environment at $4{ }^{\circ} \mathrm{C}$ in the dark until use.

Figure 1

\subsection{Measurement setup}

To optimize the assay conditions, the $\mu \mathrm{PAD}$ was used by adding $10 \mu \mathrm{L}$ of the sample or standard dropped on the sampling zone flowing through the two channels and 
reconstituting the dry reagents. After $35 \mathrm{~min}$, the device was imaged with a digital camera set as follow: macro, ISO 100, shutter speed 1/500 s, aperture value f/4 and focal length of $6.1 \mathrm{~mm}$. To keep all the image gathering conditions constant, the $\mu \mathrm{PAD}$ was placed in a fixed position in a cubic wooden homemade light box with two LED $6500 \mathrm{~K}$ lamps to digitalize[17]. The captures were obtained in JPEG format and the analysis of the region of interest (ROI) of the images was performed using Image $\mathbf{J}$ software obtaining the grey scale value $(\mathrm{g})[18]$.

The analytical parameter used is the normalized enzyme inhibition (I)[19] (eq. 1).

$$
\mathrm{I}=\frac{\mathrm{g}_{\mathrm{x}}-\mathrm{g}_{u}}{\mathrm{~g}_{\mathrm{i}}-\mathrm{g}_{\mathrm{u}}}
$$

Where $g_{x}$ is the value of $g$ coordinate for the inhibited reaction for sample or standard, $g_{u}$ is the value of $g$ coordinate for the uninhibited reaction with blank solution, and $g_{i}$ is the value of $g$ coordinate for the totally inhibited reaction.

\section{Results and discussion}

The $\mu \mathrm{PAD}$ developed for pesticide determination is based on the inhibition of the $\mathrm{AChCl}$ hydrolysis by AChE in the presence of organophosphorus and carbamate pesticides. When $\mathrm{AChCl}$ is hydrolysed, producing choline and acetic acid, the extension of the reaction is monitored using a $\mathrm{pH}$ indicator. If pesticides are present, the reaction will be inhibite, reducing the amount of acetic acid generated[20]. On the other hand, the use of capillary supports has the advantage of the possibility of including different analytical operations in the device, such as sample buffering, sample filtration, and carrying out different reactions with the device[21]. The $\mu \mathrm{PAD}$ developed for pesticide determination is easy to use and only requires the addition of sample, because all the reagents needed to perform the determination are included in the $\mu$ PAD.

\subsection{Design of $\mu P A D$ and optimization of experimental conditions}

Filter paper was chosen to serve as the support for the enzyme and substrate as well as the $\mathrm{pH}$ indicator used for colorimetric transduction because of its low cost and efficient handling of pre-treated reagents. The sensitivity of the AChE inhibition assay on paperbased microfluidic devices ( $\mu$ PAD) is affected by various factors, including enzyme and substrate concentrations, $\mathrm{pH}$ indicator, wetness and incubation time. Therefore, the optimal conditions for the $\mu$ PAD for pesticides were studied. 
As $\mathrm{pH}$ indicator, two dyes were tested, cresol red and bromocresol purple, both immobilized in sol-gel and tested at different $\mathrm{pH}$ values from 4.0 to $9.0(\mathrm{n}=3)$. In the case of cresol red, the change of colour in the range considered when it is included in the solgel was lower than expected, and it was dismissed. In the case of BP, the colour variation in the range from 4.0 to 9.0 was from yellow to violet (Figure 1). Different colour coordinates were considerer in order to quantify the colour change (RGB, HSV and grey scale), selecting the third (Figure 2) to calculate the normalized enzyme inhibition parameter I (eq. 1).

Figure 2

The BP concentrations tested in the sol-gel were from 0.1 to $0.8 \mathrm{mg} \cdot \mathrm{mL}^{-1}$. When low concentrations of BP are used, the colour developed is too weak to be used in enzymatic reactions. On the other hand, if the amount of the indicator is too high, high colour intensity develops and it is more difficult to observe the variations in colour. Therefore, $0.5 \mathrm{mg} \cdot \mathrm{mL}^{-1}$ of the $\mathrm{pH}$ indicator was selected as the optimal value for successive experiments, because it provides a colour change that can be quantified for a wider range of pesticide concentrations.

After selecting the BP as indicator, the next step in the optimization process is the immobilization of $\mathrm{AChCl}$ and $\mathrm{AChE}$, together with the $\mathrm{BP}$ on the $\mu \mathrm{PAD}$. If all the reagents are contained on the device, it is only necessary to add a sample to perform the pesticide determination but, as previously described, the mechanism used to determine the pesticide concentration is based on the inhibition of AChE. As a result, the enzyme and substrate cannot be together before adding the sample. Different strategies have been used to prevent the contact between enzyme and substrate. Some solve the problem by adding some amount of reagent and the simplest assay introduces the sample to the sensing zone of the paper device containing the enzyme and Ellman's reagent (DTNB), via dipping; after incubation, the paper is placed into acetylthiocholine iodide (ATCh) to develop the colour. Kavruk et al.[22] immobilize AChE and DTNB on filter paper and add the problem with ATCh to dry paper. Sicard et al.[23] use a wax-printed device with two zones for sensing and substrate. After dropping the sample in the sensing zone, the device must be dipped in a water bath to move the substrate, indoxyl acetate, to the sensing zone. The use of a paper-based foldable device is proposed to solve the problem. This consists of a cover and detection sheets containing indoxyl acetate and AChE, respectively. After the sample is deposited and incubated, closing the foldable device starts the reaction[24]. 
In this work, we present a device with a simplified, small design with all the reagents immobilized using a double-path $\mu$ PAD (Figure 1) composed of two transport channels separated by a gap, where $\mathrm{AChE}$ and $\mathrm{AChCl}$ are immobilized in each of the channels, and a detection zone where a $\mathrm{pH}$ indicator in sol-gel is immobilized. Therefore, the addition of the aqueous sample reconstitutes the reagents, initiating the inhibition process and transporting all reagents to the transduction area through the two channels. This strategy produces an easy-to-use device that includes all the reagents in dry state.

The AChE concentration was fixed at $1 \mathrm{U} \cdot \mathrm{mL}^{-1}$, dropping $5 \mu \mathrm{L}$ of the previously prepared solution according to section 2.3. This amount was selected because it is easy to work with and the expense for the reagent is minimal[25]. The optimal concentration of $\mathrm{AChCl}$ solution was obtained after testing different concentrations from 2 to $15 \mathrm{mM}$, by adding $1 \mu \mathrm{L} \mathrm{AChCl}$, three replicates each and adding $10 \mu \mathrm{L}$ of BSA solution on the sampling zone so that the enzyme and substrate reach the sensing area and react.

Figure 3

Figure 3 shows the signal variation depending on the $\mathrm{AChCl}$ concentration, 35 minutes after the addition. The $\mathrm{g}$ coordinate increases as the $\mathrm{AChCl}$ concentration rises, reaching a stable signal at $8 \mathrm{mM}$. For $\mathrm{AChCl}$ concentrations above this value, the enzyme reaches saturation. The concentration selected as optimal was $8 \mathrm{mM}$, because the signal is steady at that value, but a lower quantity of reagents is used.

The reaction time is one of the parameters that must be optimized when a $\mu$ PAD is developed, so that the signal measured is steady. In order to study the signal variation over time, the device's colour was recorded at $\mathrm{pH} 7.0$ with $5 \mathrm{mM}$ phosphate buffer at different reaction time $0,15,20,35$ and $65 \mathrm{~min}(\mathrm{n}=3)$. Figure 4 shows that the grey colour coordinate from the detection zone increases as the time increases, until a steady value is reached at $35 \mathrm{~min}$; this reaction time was selected as the optimum value.

Figure 4

\subsection{Analytical characterization of microfluidic device}

Once the $\mu \mathrm{PAD}$ was optimized in terms of $\mathrm{pH}$, reaction time, reagent and design, it was calibrated using carbaryl and chlorpyrifos pesticides. For this purpose, two sets of 11 and 9 standard solutions, respectively, containing the pesticides from $1 \mathrm{ng} \cdot \mathrm{L}^{-1}$ to $200 \mu \mathrm{g} \cdot \mathrm{L}^{-1}$ for carbaryl and from $5 \mathrm{ng} \cdot \mathrm{L}^{-1}$ to $200 \mu \mathrm{g} \cdot \mathrm{L}^{-1}$ for chlorpyrifos, respectively, were prepared, measuring three replicates for each (Figure 5). The data were fit to the Boltzmann equation (eq. 2) and the analytical parameters calculated are presented in Table 1. 


$$
y=A_{2}+\frac{\left(A_{1}-A_{2}\right)}{1+e^{\frac{\left(x-X_{0}\right)}{d_{x}}}}
$$

The limit of detection was calculated as 6 times the standard deviation of the blank [26], obtaining the value of $0.24 \mu \mathrm{g} \cdot \mathrm{L}^{-1}$ for carbaryl and $2.00 \mu \mathrm{g} \cdot \mathrm{L}^{-1}$ for chlorpyrifos.

The repeatability as RSD, obtained using 8 different $\mu$ PAD for $2 \mu \mathrm{g} \cdot \mathrm{L}^{-1}$ carbaryl and 15 $\mu \mathrm{g} \cdot \mathrm{L}^{-1}$ chlorpyrifos, were 4.2 and $5.5 \%$, respectively, acceptable figures considering the measuring system used.

Figure 5

\subsection{Determination of pesticides in real water samples}

To evaluate this method for quantifying AChE inhibitory effects by pesticides, recovery experiments were carried out using tap water, sea water, well water, river water, and spring water spiked with a known amount of carbaryl and chlorpyrifos (Table 3). To study the matrix effects experiments using pesticide-free water samples were performed and an average recovery of 97.7 and $102.3 \%$ for carbaryl and chlorpyrifos, respectively was obtained pooled RSD was $2.5 \%, \mathrm{~N}=5$ for each concentration value. Pesticide recoveries ranged between 85.0 and $116.0 \%$.

\section{Conclusion}

This study describes a new, simple and rapid colorimetric method for pesticide determination based on a double path $\mu \mathrm{PAD}$ which design and characterization is presented. The signal transduction is the result of substrate hydrolysis by the inhibited enzyme by pesticides, with chlorpyrifos and carbaryl as a model, which produces local $\mathrm{pH}$ alterations of a $\mathrm{pH}$ dye immobilized in sol-gel on the reaction zone. The inhibition percentage calculated from the $\mathrm{g}$ value obtained from the digitalized reaction zone is related to pesticide concentration. The double path design used allow for the separately immobilization of all needed reagents on the device easing its use. Carbaryl and chlorpyrifos were successfully determined using the proposed method with an LOD of 0.24 and $2.00 \mu \mathrm{g} \cdot \mathrm{L}^{-1}$ with a reproducibility between 4.2 and $5.5 \%$, respectively The method was successfully applied to the direct analysis of natural water samples without sample pretreatment with good recovery values.

\section{Acknowledgements}

This study was supported by a project from the Spanish MINECO (CTQ2016-78754-C21-R), a project partially supported by European Regional Development Funds (ERDF). 


\section{References}

[1] X. Yan, H. Li, X. Su, Review of optical sensors for pesticides, TrAC Trends in Analytical Chemistry 103 (2018) 1-20.

[2] A.M. Taiwo, A review of environmental and health effects of organochlorine pesticide residues in Africa, Chemosphere 220 (2019) 1126-1140.

[3] S. Chowdhary, R. Bhattacharyya, D. Banerjee, Acute organophosphorus poisoning, Clinica chimica acta; international journal of clinical chemistry 431 (2014) 66-76.

[4] M.L. Ruiz del Castillo, M. Rodriguez-Valenciano, G. Flores, G.P. Blanch, New method based on Solid Phase Microextraction and Multidimensional gas chromatography-mass spectrometry to determine pesticides in strawberry jam, LWT Food Science and Technology 99 (2019) 283-290.

[5] K. Seebunrueng, Y. Santaladchaiyakit, S. Srijaranai, Vortex-assisted low density solvent based demulsified dispersive liquid-liquid microextraction and high-performance liquid chromatography for the determination of organophosphorus pesticides in water samples, Chemosphere 103 (2014) 51-58.

[6] J.I. Cacho, N. Campillo, P. Viñas, M. Hernandez-Cordoba, In situ ionic liquid dispersive liquid-liquid microextraction coupled to gas chromatography-mass spectrometry for the determination of organophosphorus pesticides, Journal of Chromatography A 1559 (2018) 95-101.

[7] T. Tang, J. Deng, M. Zhang, G. Shi, T. Zhou, Quantum dot-DNA aptamer conjugates coupled with capillary electrophoresis: A universal strategy for ratiometric detection of organophosphorus pesticides, Talanta 146 (2016) 55-61.

[8] L.M. Fu, Y.N. Wang, Detection methods and applications of microfluidic paper-based analytical devices, TrAC Trends in Analytical Chemistry 107 (2018) 196-211.

[9] S. Deng, T. Yang, W. Zhang, C. Ren, J. Zhang, Y. Zhang, T. Cui, W. Yue, Rapid detection of trichlorfon residues by a microfluidic paper-based phosphorus-detection chip ( $\mu$ PPC), New Journal of Chemistry 43(19) (2019) 7194-7197.

[10] Z. Zhang, X. Ma, M. Jia, B. Li, J. Rong, X. Yang, Deposition of CdTe quantum dots on microfluidic paper chips for rapid fluorescence detection of pesticide 2,4-D, Analyst 144 (2019) 1282-1291.

[11] W. Chungchai, M. Amatatongchai, R. Meelapsom, K. Seebunrueng, S. Suparsorn, P. Jarujamrus, Development of a novel three-dimensional microfluidic paper-based analytical device (3D- $\mu$ PAD) for chlorpyrifos detection using graphene quantum-dot capped gold nanocomposite for colorimetric assay, International Journal of Environmental Analytical Chemistry (2019) https://doi.org/10.1080/03067319.2019.1650921.

[12] S. Nouanthavong, D. Nacapricha, C.S. Henry, Y. Sameenoi, Pesticide analysis using nanoceria-coated paper-based devices as a detection platform, Analyst 141 (2016) 1837-1846.

[13] Q.T. Hua, H. Shibata, Y. Hiruta, D. Citterio, Flow control-based 3D $\mu$ PADs for organophosphate pesticide detection, Analytical Science 35 (2019) 393-399.

[14] S. Jahanshahi-Anbuhi, P. Chavan, C.M. Sicard, V. Leung, S.M.Z. Hossain, R. Pelton, J.D. Brennan, C.D.M. Filipe, Creating fast flow channels in paper fluidic devices to control timing of sequential reactions, Lab on a Chip 12 (2012) 5079-5085.

[15] N. Yang, P. Wang, C.Y. Xue, J. Sun, H.P. Mao, P.K. Oppong, A portable detection method for organophosphorus and carbamates pesticide residues based on multilayer paper chip, Journal of Food Process Engineering (2018) https://doi.org/10.1111/jfpe.12867

[16] R. Makote, M. Collinson, Organically modified silicate films for stable pH sensors, Analytica Chimica Acta 394 (1999) 195-200.

[17] I. Perez de Vargas-Sansalvador, M.M. Erenas, D. Diamond, B. Quilty, L.F. Capitan-Vallvey, Water based-ionic liquid carbon dioxide sensor for applications in the food industry, Sensors and Actuators B 253 (2017) 302-309.

[18] L.F. Capitan-Vallvey, N. Lopez-Ruiz, A. Martinez-Olmos, M.M. Erenas, A.J. Palma, Recent developments in computer vision-based analytical chemistry: A tutorial review, Analytica Chimica Acta 899 (2015) 23-56.

[19] D. Liu, W. Chen, J. Wei, X. Li, Z. Wang, X. Jiang, A Highly Sensitive, Dual-Readout Assay Based on Gold Nanoparticles for Organophosphorus and Carbamate Pesticides, Analytical Chemistry 84 (2012) 4185-4191.

[20] M. Pohanka, J.Z. Karasova, K. Kuca, J. Pikula, O. Holas, J. Korabecny, J. Cabal, Colorimetric dipstick for assay of organophosphate pesticides and nerve agents represented by paraoxon, sarin and VX, Talanta 81 (2010) 621-624. 
[21] M.M. Erenas, B.n. Carrillo-Aguilera, K. Cantrell, S. Gonzalez-Chocano, I.M. Perez de VargasSansalvador, I. de Orbe-Paya, L.F. Capitan-Vallvey, Real time monitoring of glucose in whole blood by smartphone, Biosensors and Bioelectronics 136 (2019) 47-52.

[22] M.Kavruk, V.C.Özalp, H.A.Öktem, Portable Bioactive Paper-Based Sensor for Quantification of Pesticides, Journal of Analytical Methods in Chemistry, (20013) ID 932946.

[23] C. Sicard, C. Glen, B. Aubie, D. Wallace, S. Jahanshahi-Anbuhi, K. Pennings, G.T. Daigger, R. Pelton, J.D. Brennan, C.D.M. Filipe, Tools for water quality monitoring and mapping using paper-based sensors and cell phones, Water Research 70 (2015) 360-369.

[24] A. Apilux, C. Isarankura-Na-Ayudhya, T. Tantimongcolwat, V. Prachayasittikul, Paper-based acetylcholinesterase inhibition assay combining a wet system for organophosphate and carbamate pesticides detection, EXCLI Journal (2015) 307-319.

[25] Q. Luo, F. Yu, F. Yang, C. Yang, P. Qiu, X. Wang, A 3D-printed self-propelled, highly sensitive minimotor for underwater pesticide detection, Talanta 183 (2018) 297-303.

[26] G. Mistlberger, G.A. Crespo, E. Bakker, Ionophore-Based Optical Sensors, Annual Review of Analytical Chemistry 7 (2014) 483-512.

[27] B. Lin, Y. Yan, M. Guo, Y. Cao, Y. Yu, T. Zhang, Y. Huang, D. Wu, Modification-free carbon dots as turn-on fluorescence probe for detection of organophosphorus pesticides, Food Chemistry 245 (2018) 1176-1182.

[28] L.F. Sgobbi, S.A.S. Machado, Functionalized polyacrylamide as an acetylcholinesterase-inspired biomimetic device for electrochemical sensing of organophosphorus pesticides, Biosensors and Bioelectronics 100 (2018) 290-297.

[29] M.-G. Lee, V. Patil, Y.-C. Na, D.S. Lee, S.H. Lim, G.-R. Yi, Highly stable, rapid colorimetric detection of carbaryl pesticides by azo coupling reaction with chemical pre-treatment, Sensors and Actuators B 261 (2018) 489-496.

[30] V.G. Andreou, Y.D. Clonis, A portable fiber-optic pesticide biosensor based on immobilized cholinesterase and sol-gel entrapped bromcresol purple for in-field use, Biosensors and Bioelectronics 17 (2002) 61-69.

[31] D. Du, J. Ding, J. Cai, A. Zhang, Determination of carbaryl pesticide using amperometric acetylcholinesterase sensor formed by electrochemically deposited chitosan, Colloids and Surfaces B: Biointerfaces 58 (2007) 145-150.

[32] S.M.Z. Hossain, R.E. Luckham, M.J. McFadden, J.D. Brennan, Reagentless Bidirectional Lateral Flow Bioactive Paper Sensors for Detection of Pesticides in Beverage and Food Samples, Analytical Chemistry 81 (2009) 9055-9064. 


\section{Figure captions}

Figure 1. Picture of the $\mu \mathrm{PAD}$ for pesticides: a) Basic form of the indicator (inhibited reaction); b) Acidic form of the indicator (uninhibited reaction); c) $\mu \mathrm{PAD}$ design and dimensions.

Figure 2. Evolution of g coordinate with $\mathrm{pH}$ of sol-gel containing BP deposited on $\mu \mathrm{PAD}$. Figure 3. Optimization of $\mathrm{AChCl}$ concentration.

Figure 4. Influence of drying time on $g$ value.

Figure 5. Calibration function for both pesticides $(n=3)$. 

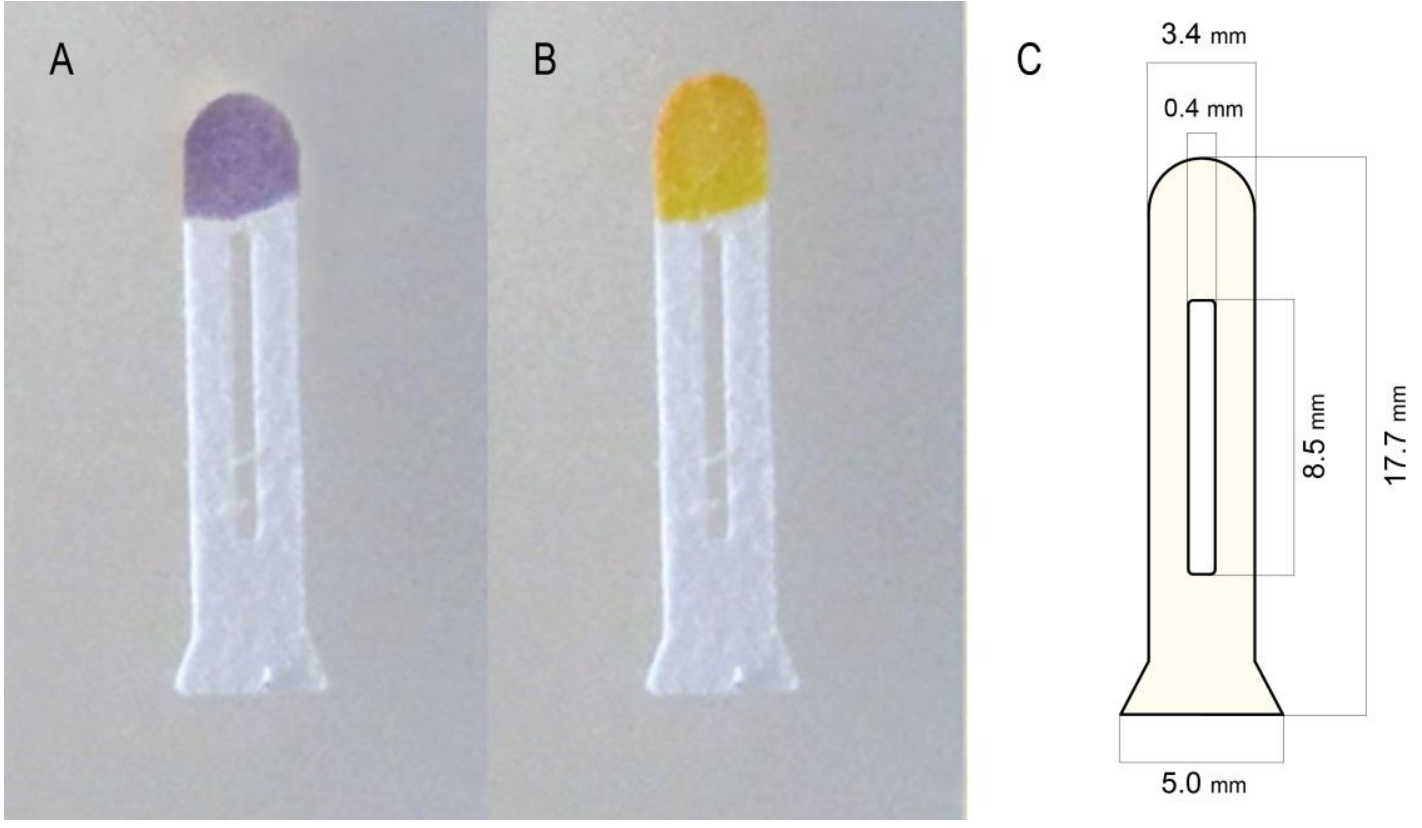

Figure 1 


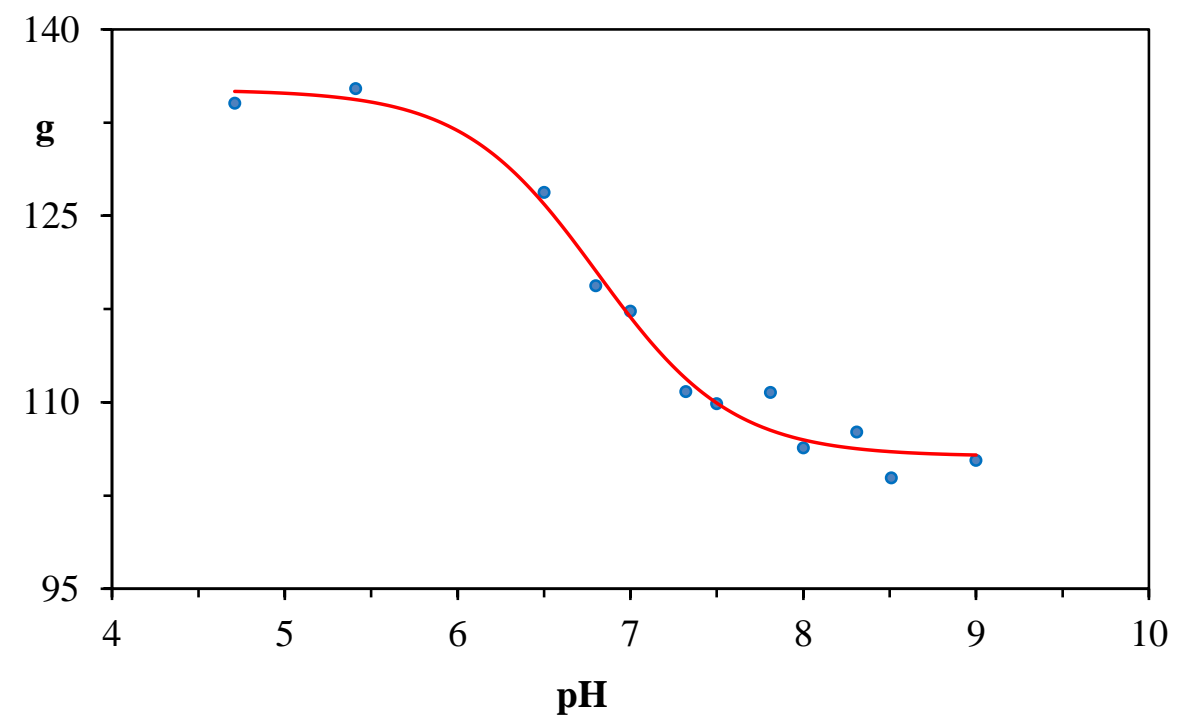

Figure 2 


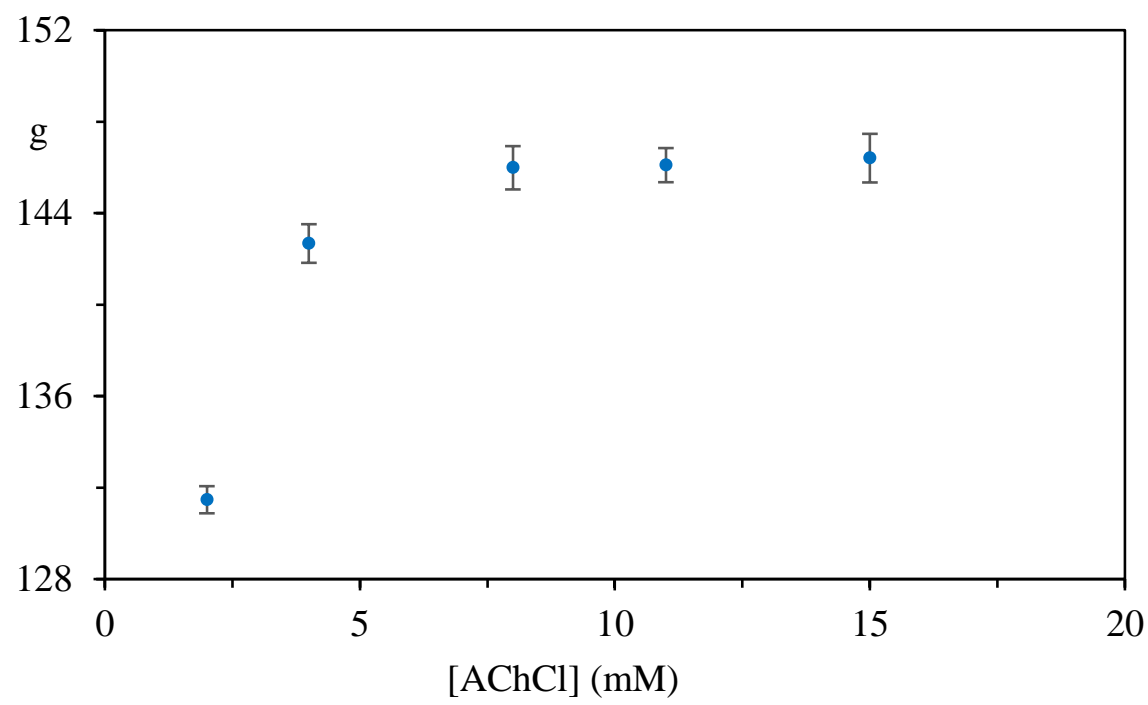

Figure 3 


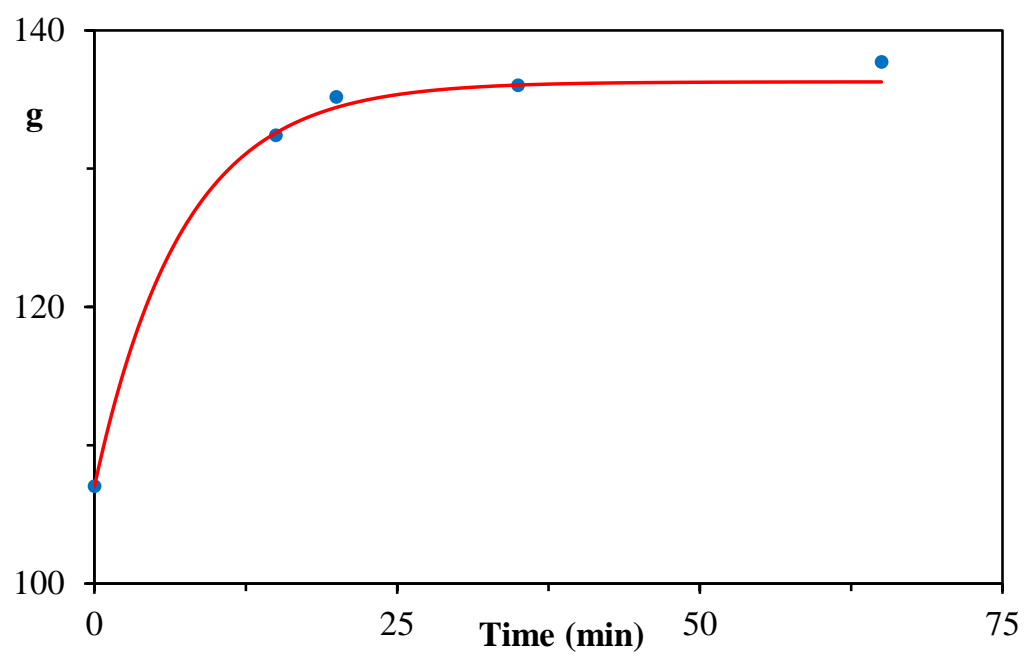

Figure 4 

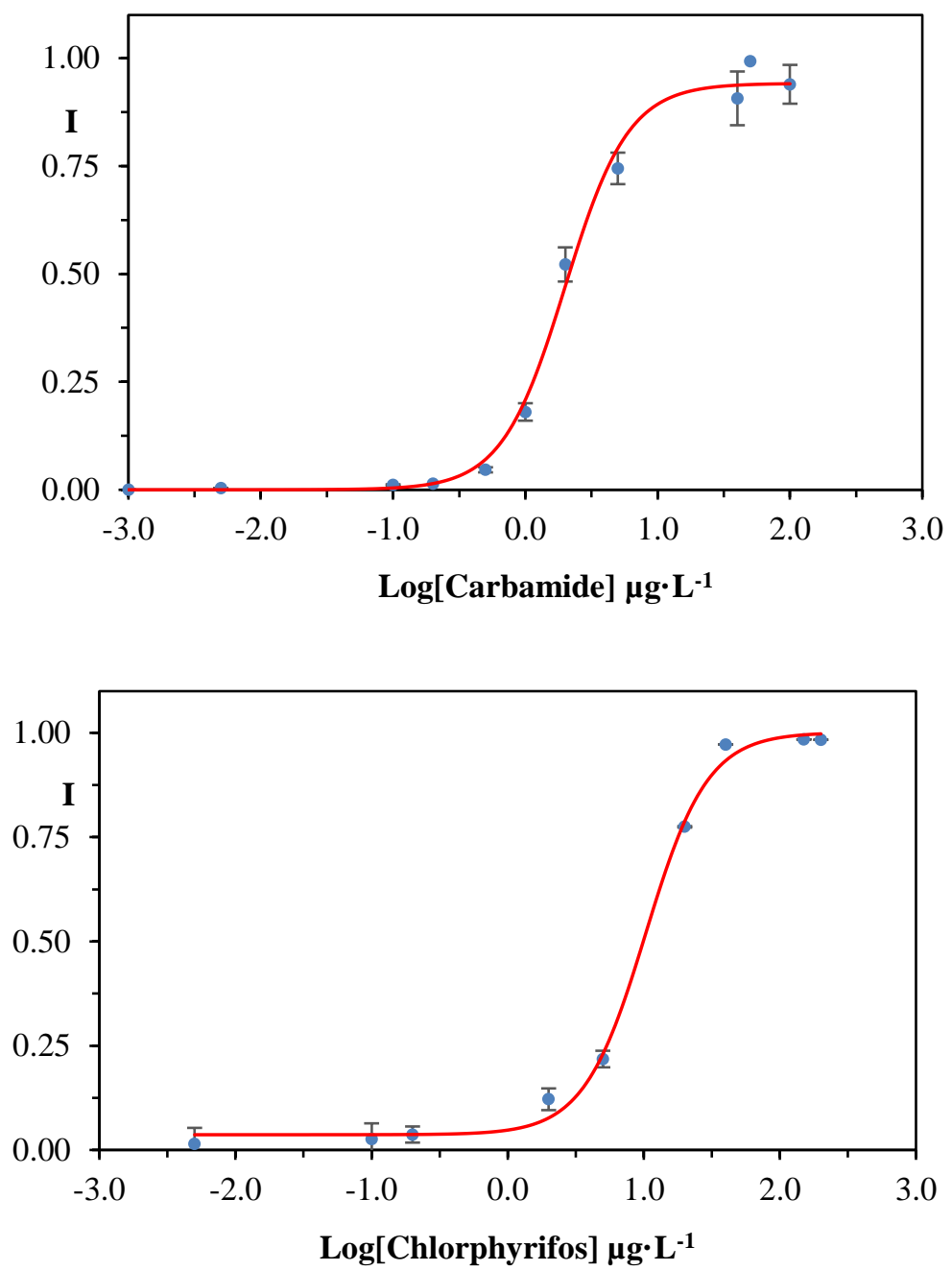

Figure 5 
Table 1. Analytical characteristics of $\mu \mathrm{PAD}$ for pesticides

\begin{tabular}{|l|c|c|}
\hline Analytical Parameters & Carbaryl & Chlorpyrifos \\
\hline Measurement range $\left(\mu \mathrm{g} \cdot \mathrm{L}^{-1}\right)$ & $0.24-20$ & $2.00-45$ \\
\hline $\mathrm{A}_{1}$ & $1.00 \cdot 10^{-4}$ & 0.0036 \\
\hline $\mathrm{A}_{2}$ & 0.9415 & 1.0010 \\
\hline $\mathrm{X}_{0}$ & 0.3010 & 1.0139 \\
\hline $\mathrm{dx}$ & 0.2395 & 0.2275 \\
\hline $\mathrm{R}^{2}$ & 0.9930 & 0.9953 \\
\hline $\mathrm{LOD}\left(\mu \mathrm{g} \cdot \mathrm{L}^{-1}\right)$ & 0.24 & 2.00 \\
\hline Precision $(\mathrm{RSD} \%) 200 \mu \mathrm{g} \cdot \mathrm{L}^{-1}$ & 4.2 & 5.2 \\
\hline Precision $(\mathrm{RSD} \%) 500 \mathrm{mg} \cdot \mathrm{L}^{-1}$ & 4.5 & 5.5 \\
\hline
\end{tabular}


Table 2. Comparison of the present assay with other reported methods for the detection of pesticides

\begin{tabular}{|c|c|c|c|c|c|c|}
\hline Probe & Measurement & Pesticide & Lineal range & $\begin{array}{l}\text { RSD } \\
(\%)\end{array}$ & LOD & Ref. \\
\hline Carbon dots & Fluorescence & Chlorpyrifos & $\begin{array}{c}0.01-1.0 \mu \mathrm{g} \cdot \\
\mathrm{mL}^{-1}\end{array}$ & 5 & $3 \mathrm{ng} \cdot \mathrm{mL}^{-1}$ & {$[27]$} \\
\hline Polyacrylamide & Electrochemistry & Chlorpyrifos & $1.0-10.0 \mu \mathrm{g} \cdot \mathrm{L}^{-1}$ & 11.7 & $\begin{array}{c}0.83 \\
\mu \mathrm{mol} \cdot \mathrm{L}^{-1}\end{array}$ & {$[28]$} \\
\hline RB-AuNPs & $\begin{array}{c}\text { Colorimetric and } \\
\text { Fluorescence }\end{array}$ & Carbaryl & $0.4-3.0 \mu \mathrm{g} \cdot \mathrm{L}^{-1}$ & $5-8$ & $0.23 \mu \mathrm{g} \cdot \mathrm{L}^{-1}$ & [25] \\
\hline $\begin{array}{l}\text { Azo-coupling } \\
\text { reaction }\end{array}$ & Colorimetric & Carbaryl & $50-500 \mu \mathrm{M}$ & - & $50 \mu \mathrm{M}$ & [29] \\
\hline Fiber-optic & Colorimetric & Carbaryl & $0.11-8 \mathrm{mg} \cdot \mathrm{L}^{-1}$ & $3-5$ & $108 \mu \mathrm{g} \cdot \mathrm{L}^{-1}$ & {$[30]$} \\
\hline $\begin{array}{c}\text { AChE-Chitosan- } \\
\mathrm{Au}\end{array}$ & Electrochemistry & Carbaryl & $\begin{array}{l}0.005-0.1 \\
\mu \mathrm{g} \cdot \mathrm{mL}^{-1}\end{array}$ & - & $\begin{array}{c}0.003 \\
\mu \mathrm{g} \cdot \mathrm{mL}^{-1}\end{array}$ & [31] \\
\hline Paper sensor & Colorimetric & Carbaryl & - & - & $10 \mathrm{nM}$ & [32] \\
\hline Paper sensor & Colorimetric & $\begin{array}{c}\text { Carbaryl } \\
\text { Chlorpyrifos }\end{array}$ & $\begin{array}{l}0.24-20 \mu \mathrm{g} \cdot \mathrm{L}^{-1} \\
2.00-45 \mu \mathrm{g} \cdot \mathrm{L}^{-1}\end{array}$ & $\begin{array}{l}4.2 \\
5.2\end{array}$ & $\begin{array}{l}0.24 \mu \mathrm{g} \cdot \mathrm{L}^{-1} \\
2.00 \mu \mathrm{g} \cdot \mathrm{L}^{-1}\end{array}$ & $\begin{array}{c}\text { Current } \\
\text { study }\end{array}$ \\
\hline
\end{tabular}


Table 3. Pesticide recovery in spiked water samples

\begin{tabular}{|c|c|c|c|c|c|c|}
\hline \multirow[b]{2}{*}{$\begin{array}{l}\text { Water } \\
\text { Sample }\end{array}$} & \multirow{2}{*}{$\begin{array}{c}\text { Carbaryl } \\
\operatorname{added}\left(\mu g \cdot L^{-1}\right)\end{array}$} & \multicolumn{2}{|c|}{ $\mu$ PAD } & \multirow{2}{*}{$\begin{array}{c}\text { Chlorpyrifos } \\
\text { added } \\
\left(\mu \mathrm{g} \cdot \mathrm{L}^{-1}\right)\end{array}$} & \multicolumn{2}{|c|}{$\mu \mathrm{PAD}$} \\
\hline & & Found & Recovery (\%) & & Found & Recovery (\%) \\
\hline \multirow[b]{2}{*}{ Sea } & 10 & $11.6 \pm 0.1$ & 116.0 & 10 & $9.6 \pm 0.1$ & 91.4 \\
\hline & 2.5 & $2.4 \pm 0.1$ & 98.0 & 35 & $28.6 \pm 0.4$ & 92.6 \\
\hline \multirow[b]{2}{*}{ Well } & 10 & $9.9 \pm 0.1$ & 99.0 & 10 & $11.2 \pm 0.1$ & 91.0 \\
\hline & 2.5 & $2.4 \pm 0.1$ & 99.2 & 35 & $36.5 \pm 0.1$ & 101.0 \\
\hline \multirow[b]{2}{*}{ River } & 10 & $11.0 \pm 0.1$ & 110.4 & 10 & $11.2 \pm 0.1$ & 102.0 \\
\hline & 2.5 & $2.6 \pm 0.1$ & 113.0 & 35 & $37.1 \pm 0.3$ & 108.0 \\
\hline \multirow[b]{2}{*}{ Spring } & 10 & $10.1 \pm 0.2$ & 102.0 & 10 & $8.6 \pm 0.1$ & 85.0 \\
\hline & 2.5 & $2.6 \pm 0.1$ & 99.4 & 35 & $36.2 \pm 0.3$ & 101.0 \\
\hline \multirow[b]{2}{*}{ Rain } & 10 & $9.8 \pm 0.1$ & 99.4 & 10 & $11.4 \pm 0.1$ & 102.1 \\
\hline & 2.5 & $2.7 \pm 0.2$ & 100.0 & 35 & $33.8 \pm 0.5$ & 98.0 \\
\hline \multirow[b]{2}{*}{ Tap } & 10 & $9.2 \pm 0.1$ & 92.0 & 10 & $10.3 \pm 0.1$ & 100.4 \\
\hline & 2.5 & $2.4 \pm 0.1$ & 99.0 & 35 & $34.1 \pm 0.2$ & 99.6 \\
\hline
\end{tabular}

